Itinéraires Itinéraires

Littérature, textes, cultures

\title{
Derek Jarman : le peintre à la caméra
}

\section{Nicole Cloarec}

\section{OpenEdition}

Journals

Édition électronique

URL : http://journals.openedition.org/itineraires/2414

DOI : $10.4000 /$ itineraires. 2414

ISSN : 2427-920X

Éditeur

Pléiade

Référence électronique

Nicole Cloarec, «Derek Jarman : le peintre à la caméra », Itinéraires [En ligne], 2014-2 | 2015, mis en ligne le 16 juin 2015, consulté le 02 octobre 2020. URL : http://journals.openedition.org/itineraires/ 2414 ; DOI : https://doi.org/10.4000/itineraires.2414

Ce document a été généré automatiquement le 2 octobre 2020.

\section{(c) (i) (9)}

Itinéraires est mis à disposition selon les termes de la licence Creative Commons Attribution - Pas d'Utilisation Commerciale - Pas de Modification 4.0 International. 


\title{
Derek Jarman : le peintre à la caméra
}

\author{
Nicole Cloarec
}

\section{NOTE DE L'AUTEUR}

Cet article reprend en partie mon article « Derek Jarman : lyrisme et radicalité » (Cloarec 2010 : 202-209).

1 Derek Jarman est sans conteste devenu une figure majeure du cinéma britannique des années 1970 et 1980 et son influence perdure à travers par exemple son actrice fétiche Tilda Swinton et des metteurs en scène comme John Maybury, Isaac Julien ou encore Sally Potter. Cependant, comme le rappelle Chrissie Iles dans le catalogue de l'exposition organisée à la Serpentine Gallery de Londres en 2008, il serait injuste et inexact de ne se souvenir que de Jarman cinéaste (Iles 2008 : 64-76). Dans une interview très émouvante donnée au British Council juste avant sa mort du sida en 1994, Derek Jarman déclarait d'ailleurs humblement qu'il ne se considérait pas comme un cinéaste mais simplement comme quelqu'un qui a toujours peint et qui a fait des films ${ }^{1}$. Surtout, Jarman fut un artiste polyvalent ${ }^{2}$ qui refusait toute idée de hiérarchie en art, reprenant à son compte la devise d'un de ses mentors, le cinéaste Michael Powell : «all arts are one »(«tous les arts ne font qu'un »).

2 En quoi le cas Jarman peut-il nous aider à penser les rapports entre peinture et cinéma ? Et, tout d'abord, de quelle peinture et de quel cinéma parle-t-on?

3 L'œuvre picturale comme filmique de Jarman est traversée par deux caractéristiques fondamentales : d'abord son engagement militant contre toutes les discriminations et en particulier celles touchant les homosexuels, ensuite son indépendance vis-à-vis de toute logique commerciale. Jarman aimait à rappeler que ses huit premiers longs métrages n'avaient pas coûté plus d'un million de livres (cité dans Lippard $1996: 2$ ) et il a magistralement retourné la contrainte des budgets très réduits en une source de liberté créatrice, exprimant sa vision personnelle d'artiste sans faire de compromis. 
Pour Jarman, l'art est important parce qu'il permet d'exprimer les expériences personnelles les plus intimes en résonance avec des questions sociales et politiques plus larges.

4 Après des études d'histoire et de littérature à l'université de Londres, Jarman suit une formation de peintre de 1963 à 1967 à la Slade School of Art où il rencontre David Hockney et Patrick Procktor. C'est d'ailleurs le succès de David Hockney qui l'amène à se détourner de l'esthétique du pop art alors en vogue, ainsi que de la peinture figurative. Son œuvre picturale peut se diviser en trois grandes périodes. Les premières toiles, exécutées après ses années à slade, représentent des paysages austères, géométriques et minimalistes; le deuxième groupe d'œuvres, produites dans les années 1980, est centré autour d'une série de toiles «noires », grattées ou incrustées d'objets divers; enfin la troisième et dernière période de création plastique est étroitement liée à l'avancée de sa maladie et inclut de nouveau des techniques de collage ainsi que des toiles noires, mais aussi de grands tableaux proches de l'expressionnisme abstrait qui exhibent de rageuses explosions de couleurs.

5 Jarman, doté d'une vaste connaissance en histoire de l'art et d'une insatiable curiosité, reconnaît volontiers les nombreuses influences qui ont façonné son œuvre, de William Blake (inspirant ses décors de The Rake's Progress de Stravinsky mis en scène par Ken Russell au Pergola de Florence, en 1982) à Paul Nash, Giorgio de Chirico et René Magritte (dont l'influence apparaît dans ses premières toiles de paysages géométriques et désertiques), Ad Reinhardt (pour ses noirs «espagnols» des années 1980), Kurt Schwitters, Joseph Cornell et Robert Rauschenberg dont l'impact est perceptible dans ses collages, et bien sûr Le Caravage et Yves Klein : la liste des noms célèbres est longue. Mais ce qui importe le plus, c'est bien entendu l'appropriation des techniques à des fins personnelles et l'exploration des questions esthétiques posées par ces peintres. À l'instar de son œuvre picturale évoluant de recherches minimalistes vers une esthétique expressionniste et rageuse, l'œuvre cinématographique de Jarman semble divisée entre ces deux tendances apparemment opposées, qu'exacerbe encore la diversité des supports utilisés : des films expérimentaux en super 8 ou $16 \mathrm{~mm}$ aux longs métrages de facture plus classique, tournés en $35 \mathrm{~mm}$ et bénéficiant d'une sortie en salle.

6 Mon propos est de montrer en quoi ces deux tendances sont constitutives d'une même poétique cinématographique résolument anti-réaliste, que caractérise un même souci d'explorer les limites de la représentation. Afin d'explorer la manière dont la problématique picturale imprègne les films, je me concentrerai en particulier sur deux questions fondamentales qui traversent toute l'histoire de l'art: d'une part celle de la dichotomie entre surface et profondeur, d'autre part celle d'une tension entre figuration et défiguration. Or dans les deux cas, la question se pose en termes filmiques par le traitement de la lumière et des couleurs.

7 On ne compte plus les déclarations de cinéastes cherchant à ancrer le septième art dans une histoire picturale plus ancienne et la tentation est forte d'y effectuer une lecture à rebours, selon ce qu'Eisenstein appelait le "cinématisme » et que Jacques Aumont définit comme "la rétroaction conceptuelle et analytique du cinéma sur les arts traditionnels » (Aumont 1989 : 224). À la suite d'un Godard écrivant dans ses Histoire(s) du cinéma qu'« avec Édouard Manet commence la peinture moderne, c'est-à-dire le cinématographe " (Godard 2006 : 55) ou d'un Greenaway affirmant que Vermeer fut le premier cinéaste, Jarman a déclaré que si Le Caravage avait vécu à notre époque, il 
aurait jeté ses pinceaux dans le Tibre et se serait procuré... une caméra vidéo ! Jarman 1984 : 9-10).

8 Au-delà de la boutade, la référence au Caravage est déterminante de plusieurs points de vue. Dans Caravaggio (1986), Jarman évoque la vie du peintre à travers son œuvre et, ce faisant, y dresse un autoportrait en creux, suggérant que l'œuvre s'est nourrie de la vie, et qu'à l'inverse l'artiste n'a vécu pleinement qu'à travers l'expressivité de ses toiles. Le film explore et s'approprie la radicalité de l'artiste dans sa conception de l'espace, à savoir qu'il est fondé sur des compositions frontales, à rebours d'une narration basée sur le suspense et la progression linéaire ${ }^{3}$.

9 Ainsi les nombreuses citations picturales ${ }^{4}$, cet « effet de pure surface » (Aumont 1989: 73), dont parle Aumont pour qualifier un rapport somme toute superficiel entre peinture et cinéma, opèrent moins dans la recherche d'une reconstitution parfaite, même si on ne peut manquer d'être frappé par l'exactitude des similitudes, que dans la volonté d'imposer le temps suspendu de la scène peinte au temps du film. C'est donc moins l'effet tableau ou l'effet cadre qui entre en jeu que la dramatisation du regard. Ce n'est certainement pas un hasard si la toute première œuvre citée est la Tête de Méduse $e^{5}$ : la tête tranchée du monstre au regard pétrifiant signe par sa défaite la victoire de la vision sur l'impossible à voir, le cadre circulaire renvoyant directement au regard du spectateur. Le plus souvent, le film s'ingénie à dramatiser le regard sur l'œuvre en instaurant une triangulation des regards entre le peintre - et à travers lui le spectateur -, son modèle et son œuvre. Ainsi, pour évoquer L'Amour vainqueur ${ }^{6}$, le plan filmique est partagé en deux espaces distincts : au premier plan la toile y apparaît de biais, comme pour souligner les décalages de sa reconstitution au second plan (le chérubin nu devient une jeune contorsionniste vêtue, la sphère bleue étoilée à peine perceptible dans l'original est placée en évidence). La toile devient ainsi une mise en chorégraphie mais surtout elle est prise dans le jeu de regard complice qui s'instaure entre le peintre, son modèle et le personnage de son œuvre.

À plusieurs reprises, la mise en scène brouille ainsi les distances entre espaces hétérogènes, et instaure deux mouvements contradictoires: la mise en espace de l'œuvre peinte au travers de modèles vivants d'une part, l'intégration de l'artiste au sein de sa toile d'autre part, lorsque celui-ci est filmé en plan frontal devant la toile ${ }^{7}$ qui emplit l'écran, produisant un effet d'aplanissement de la perspective. En un clin d'œil à l'autoportrait du Caravage qui apparaît à l'arrière-plan du Martyre de saint Matthieu 8 , dans le film, le peintre occupe un espace intermédiaire entre le modèle et sa représentation, espace indéfini participant un peu des deux autres.

11 Ce questionnement de l'espace s'effectue bien sûr aussi et peut-être surtout par la lumière. Le film rend hommage à l'« inventeur » du clair-obscur, cet effet de lumière directionnelle et contrastée qui dramatise l'action sur fond obscur et qui, au même titre que l'«effet Rembrandt», est devenu une source d'inspiration pour nombre de directeurs de la photographie. Comme le rappelle Jacques Aumont, « le cinéma n'a eu de cesse de trouver des sources directionnelles » $(1989: 176)$ et c'est incontestablement dans la peinture, ténébriste ou luministe, que le cinéma a puisé ses sources d'inspiration. Mais n'en déplaise à Moholy-Nagy pour qui le cinéma doit se distinguer de la peinture en abandonnant le pigment pour la lumière (cité dans Passuth 1984 : 314-319), Caravaggio pense précisément la lumière en relation avec le pigment, interrogeant sans cesse les notions mêmes de surface et de profondeur. Dès le générique d'ouverture, un écran noir accueille conventionnellement les lettres 
blanches du titre mais on distingue nettement des traits verticaux de pinceaux. Or, au deuxième plan, une main apparaît munie d'un pinceau qui, opérant horizontalement, fait disparaitre ces traits. Puis le geste du pinceau devient vertical et refait apparaître les traces de pigment : à plusieurs reprises, la main vient repeindre la surface noire selon des coups de pinceau alternativement verticaux et horizontaux, accentuant dans le premier cas la luminosité du pigment noir, alors qu'elle annule cette luminosité dans le second. Dans un renversement ironique, le plan oscille ainsi de l'écran à la toile où l'écran se donne comme surface lisse et la toile comme épaisseur.

12 À l'image de cet insert sur le doigt de l'artiste prolongeant la main qu'il vient de peindre, la confrontation des médiums filmique et pictural souligne la contradiction inhérente à chacun d'eux, construits tous deux sur des modes de perception a priori incompatibles, « l'espace tridimensionnel représenté y étant mis sur un pied d'égalité avec l'espace, réellement bidimensionnel, de la toile» (Aumont 1989: 141). Caravaggio apporte de fait une attention toute particulière aux textures des surfaces filmées :

- Textures des décors pourtant minimalistes, le film, tourné exclusivement en studio, se déroulant dans un décor quasi unique, du fait d'un budget très réduit ${ }^{9}$. Jarman, assisté de son directeur de la photographie Gabriel Beristain et de son directeur artistique Christopher Hobbs, réussit à évoquer des atmosphères très différentes correspondant à différents lieux grâce aux variations d'éclairages et de couleurs, tels les murs blanc cassé de la pièce à Porto Ercole où meurt le peintre, ceux verdâtres de l'atelier du jeune Caravaggio, ceux bruns de l'atelier du peintre adulte ou encore ceux orangés des rues de Rome.

- Textures des épidermes, mis en valeur par l'éclairage en clair-obscur, rendant hommage au peintre qui a revitalisé l'iconographie religieuse par des modèles issus du peuple, burinés et couverts de crasse. Si l'iconographie religieuse est si présente dans l'œuvre filmique de Jarman, dès son premier long métrage Sebastiane (1976) consacré à la figure du martyr, elle est à l'évidence détournée au service du message politique et provocateur du cinéaste qui voit dans la figure du Christ un double de l'artiste et des minorités - essentiellement homosexuelles - persécutées ${ }^{10}$. Mais elle n'en est pas moins un hommage sincère à toute l'histoire picturale occidentale qui, à travers la question de l'incarnation, pose celle de la croyance en l'image.

13 La référence à saint Thomas sert ici de mise en abyme au fantasme d'une vision tactile : distance du regard contre proximité du toucher, c'est encore une fois à travers la tension entre surface et profondeur que cette dialectique se traduit sur la toile comme sur l'écran. Le visage est ainsi autant sujet aux variations chromatiques prolongeant la quête obsessionnelle de l'incarnat qu'il est traité comme toile destinée à être peinte, comme le visage de Ranuccio que Caravaggio recouvre de son sang, celui des deux amants dans The Garden (1990) entachés de Marmite, cette pâte un peu visqueuse typiquement anglaise qui évoque le sang de bœuf, ou encore les visages d'Amyl Nitrite (interprétée par Jordan) dans Jubilee (1978) ou d'Ottoline Morrell (Tilda Swinton) dans Wittgenstein (1993).

14 Sans doute est-ce dans The Last of England (1987) que Jarman nous livre le commentaire le plus réflexif sur cette dialectique entre surface et profondeur qui traverse son œuvre et anime le va-et-vient entre peinture et film. On y voit l'artiste lui-même, plongé dans une grisaille bleutée, en train de peindre l'une des toiles de sa "Caravaggio Suite", élaborée en dialogue avec le film qu'il consacre au peintre. Une série de gros plans isole une couche très épaisse de peinture noire dans laquelle Jarman insère une douille et une ampoule de verre cassée. Mais cette activité est entrecoupée, en montage parallèle, 
par une scène d'excavation archéologique où un homme, revêtu d'un manteau blanc, exhume au pinceau une grosse ampoule ainsi qu'un morceau de pellicule. Au final, le geste même de la représentation renvoie à la matérialité du médium, la métaphore de l'enfouissement à la surface de la pellicule.

Si dans Caravaggio, le noir n'apparait jamais comme absence, de lumière et de couleurs, mais comme texture ${ }^{11}$, les fonds obscurs d'Edward II (1991) et Wittgenstein, l'avantdernier film de Jarman, et le dernier tourné avec des acteurs, eux, sont totalement noirs, annihilant toute notion de décor : l'espace y devient une scène - au sens théâtral du terme - abstraite, sans même la perspective élémentaire qu'offrirait la vision de murs. En contraste, les personnages évoluent en habits de couleurs saturées - selon la trinité du noir, du blanc et du rouge sang dans Edward II, selon les trois couleurs primaires, dans Wittgenstein. Dans ce dernier film, la pensée du philosophe éponyme, connu pour sa méfiance à l'égard de l'idée même de "profondeur ", notamment dans l'usage métaphorique du langage, sert de principe esthétique. De façon ironique, alors que le film évoque le goût du philosophe pour le cinéma, on peut voir le jeune Wittgenstein, de face, portant des lunettes aux verres colorés par des filtres interférentiels, destinées à la vision en relief. Or si le dispositif de la projection est révélé comme tel au plan suivant, lorsque le jeune garçon s'amuse à intercepter de sa main le faisceau lumineux, les ombres chinoises, elles, restent résolument absentes $d u$ plan. De même, dans The Garden, Jarman a recours à de très nombreuses transparences, ce vieux procédé qui consiste à projeter des images (préalablement filmées) dans un studio, à l'aide d'un projecteur placé derrière un écran translucide. Or ces transparences ne cherchent aucunement à créer l'illusion d'une réelle coexistence des acteurs dans ces décors qui se révèlent pour ce qu'ils sont : des toiles de fond, d'autant que les paysages choisis sont le plus souvent filmés en monochrome, accentuant l'arbitraire d'une couleur devenue autonome, indifférente au réel, de même que leur dimension de surface plane.

Dans chacun de ces films, Jarman n'a de cesse d'exposer les limites mêmes d'une recherche de profondeur, récusant toute conception d'une caméra comme "fenêtre ouverte sur le monde " et il entend ainsi travailler comme un peintre le grain de ses films. Le choix, dicté par les contraintes budgétaires, de tourner en vidéo, en Super 8 ou en $16 \mathrm{~mm}$ devient propice à des recherches formelles sur la texture même du médium filmique. Toute la batterie des techniques utilisées au tournage et au montage témoigne d'une recherche quasi expérimentale sur l'image: effets grossiers de transparences cités plus haut, extérieurs filmés à travers des filtres colorés, filés et zooms rapides, ralentis et accélérés, etc. Surtout, dans The Last of England, The Garden ou encore War Requiem (1989), Jarman juxtapose les supports différents (Super $8,16 \mathrm{~mm}$, images d'archive ou vidéo) qu'il traite indifféremment soit en monochrome bleuté, rouge ou sépia, soit en noir et blanc, soit en polychromie, soulignant à chaque fois une texture particulière. Pour ce faire, Jarman transfère toutes ses images sur vidéo au montage, ce qui lui permet d'utiliser les outils de la palette graphique (Paintbox) avant de «regonfler» le tout en $35 \mathrm{~mm}$. De plus, le cinéaste utilise abondamment les surimpressions et le filmage répété des mêmes images qui perdent ainsi en définition. En conséquence, la couleur déborde des contours de l'objet, se détache d'un support matériel pour devenir tache, elle « semble s'arracher aux objets pour devenir aventure singulière » (Aumont 1989 : 215) alors que le grain de la vidéo suggère la texture de la matière picturale. 
17 À propos de In the Shadow of the Sun (1981), moyen métrage de 50 minutes, pour lequel Jarman surimpose jusqu'à sept couches d'images qu'il re-filme à chaque fois, le cinéaste évoque les toiles impressionnistes :

Les images de In the Shadow of the Sun sont saturées de rouges écarlates, d'oranges et de roses. La dégradation provoquée par les multiples re-filmages des images leur donne une aura de mystère et une énergie qui rappellent les Nymphéas de Monet ou ses meules de foin au crépuscule. (Jarman $1984: 124)$

\section{Michael O'Pray commente :}

Grâce au re-filmage et à la vitesse de prise de vue d'origine, Jarman a pu obtenir un contrôle de l'image qui produit une intense texture picturale et une pulsion rythmique. Le grain et l'effet de traînées colorées sont comme de larges coups de pinceau vigoureux et, en vertu de l'effet de dégradation dû aux re-filmages successifs, les couleurs sont souvent adoucies et forment des halos. (O'Pray $1985: 9$ )

19 Le dernier film de Jarman, Blue (1993), réalisé quelques mois avant sa mort, pousse cette négation du figuratif dans les retranchements de l'expérience limite : celle d'un écran où le visuel est réduit à un monochrome bleu et où le lieu du film est déplacé dans sa bande-son, ou plutôt dans l'abandon d'un lieu propre au film, étant donné la qualité acousmatique de la bande sonore, détachée d'une quelconque source visuelle ${ }^{12}$. Bien que le terme " Blue », dans le texte, renvoie aussi bien à la couleur qu'à une humeur, un concept, un objet ou même à une personne qui reste indéfinie, c'est avant tout en hommage à Yves Klein que Jarman a choisi la couleur de son monochrome filmique ${ }^{13}$. À la suite du peintre français, il voit dans le monochrome l'essence même de la peinture, enfin libérée des contraintes figuratives et parvenue à un état de pureté transcendantale. De façon paradoxale, on rejoint ici la question de l'incarnation en tant que matérialisation du spirituel: "Blue is darkness made visible» («le bleu, c'est l'obscurité rendue visible»). Jarman, en faisant directement référence à l'oxymore miltonien, se place de façon explicite dans la lignée des romantiques pour qui la « vision du visionnaire » prime sur le visuel.

20 À l'inverse, il arrive que l'informel se reconfigure. Dans The Angelic Conversation (1985), mise en images de quelques sonnets de Shakespeare et filmé selon une technique prisée par Jarman de trois ou six images par seconde qui, projetées à la même vitesse, donnent l'effet d'un défilement rapide de diapositives, on peut voir en très gros plans des flammes de bougie que le mouvement extrêmement ralenti transforme en taches abstraites de couleurs sur fond noir. Au comble de l'abstraction, il arrive pourtant qu'une figuration resurgisse, comme cette flamme blanche qui évoque l'essor d'une colombe, mais cette figuration n'a plus rien à voir avec le réel profilmique. Incidemment, les effets de ralenti et de saccades font du temps même un matériau plastique, nous forçant à suspendre notre regard, rendant notre perception du monde problématique, non plus acquise mais comme résultat d'un processus d'immersion.

Dans ses courts et moyens métrages, dans The Last of England ou The Garden, la nature est ainsi soumise à l'instabilité des filtres colorés : ciel et mer sont tour à tour bleus, rouges, violets, jaunes ou oranges. Jacques Aumont écrit dans L'Eil interminable : «Un coucher de soleil peint est une impression; filmé, il devient vite insupportable chromo" (Aumont 1989: 167). Jarman ose les chromos, à rebours de la remarque de Jacques Aumont sur cette réticence du cinéma à user de sa "supériorité » à réaliser sans peine les effets les plus recherchés de la peinture, à savoir la représentation de l'invisible, de l'impalpable: "Figurer l'air, l'atmosphère, et [...] la lumière, rendre compte de ces fluides mystérieux a été l'une des hantises de la peinture " (Aumont 
1989 : 234). Jarman se place ici dans la lignée des impressionnistes et du peintre anglais William Turner que le critique Michael o'Pray évoque en analysant la palette des bleus nuit, des violets et des oranges incandescents de The Last of England (O'Pray 1996 : 156).

Surtout, les effets de filés et de zoom, le jeu sur les vitesses de défilement " aberrants » (Deleuze 1985 : 53) - ralenti ou accéléré - sont mis au service d'une recherche d'effets de lumière extrêmes. L'incipit de The Garden est ici révélateur: Jarman choisit de commencer son film de façon réflexive en filmant une scène de tournage mais cette information n'apparaît clairement qu'à travers la bande-son; la scène nocturne, elle, reste à peine perceptible, n'offrant aucun repère visuel en dehors de points lumineux : on est proche ici de l'art cinétique où la lumière est filmée comme matière picturale. Jarman aime filmer les sources de lumière, torches et flammes, aussi bien de manière frontale qu'à travers les écrans de fumée qu'elles dégagent. Mais le procédé le plus caractéristique, que Jarman utilise de façon récurrente, consiste à filmer frontalement un miroir qu'une personne tient face à la caméra, provoquant par intermittence l'aveuglement du spectateur. Art of Mirrors (1973) est presque entièrement composé de cette figure; The Angelic Conversation la reprend abondamment, et on en trouve des exemples isolés dans les longs métrages, comme le miroir que tient Ariel dans Jubilee ou le prisme que tient le Martien, en référence à Newton et à la supposée transparence du monde, à la toute fin de Wittgenstein. La lumière, condition même de visibilité, devient source d'opacité. L'écran, surface de réception, se transforme alors en foyer, espace d'irradiation qui manifeste une résistance à la figuration. Leo Bersani et Ulysse Dutoit comparent d'ailleurs ces procédés de réduction du champ visuel aux tableaux de Turner et à leurs «rideaux de blancheur quasi absolue» (Bersani et Dutoit 1999: 59). Les deux critiques en concluent : Jarman «nous aveugle non pas pour rediriger notre regard, mais pour exprimer son pouvoir de metteur en scène, jusque dans sa dimension nihiliste » (Bersani et Dutoit 1999 : 59).

À l'instar de son œuvre picturale, l'œuvre cinématographique de Jarman semble divisée entre deux tendances apparemment opposées: celle de l'épure théâtrale mono- ou bichromatique et celle de l'outrance, parfois kitsch, des surimpressions et des montages ultrarapides, et ceci, aussi bien de façon diachronique qu'au sein d'une même œuvre. Or, le détour par la problématique picturale - à savoir la dichotomie entre surface et profondeur, la tension entre figuration et défiguration et le traitement conjoint de la lumière et des couleurs - nous montre combien ces deux tendances sont constitutives d'une même poétique cinématographique, résolument anti-réaliste et soucieuse d'explorer les limites de la représentation, oscillant entre un désir d'appréhension haptique du monde d'une part et une aspiration à l'abstraction d'autre part. Dans les deux cas, le film est appréhendé comme support pictural à modeler-et non simplement comme support photographique à imprimer. 


\section{BIBLIOGRAPHIE}

Aumont, Jacques, 1989, L'Æil interminable. Cinéma et peinture, Paris, Séguier.

Bersani, Leo et Dutoit, Ulysse, 1999, Caravaggio, Londres, British Film Institute, coll. « BFI Modern Classics ».

Cloarec, Nicole, 2010, « Derek Jarman : lyrisme et radicalité », Ligeia, nº 97-98-99-100, p. 202-209.

Deleuze, Gilles, 1985, Image-temps, Paris, Minuit.

Godard, Jean-Luc, 2006, Histoire(s) du cinéma, Paris, Gallimard.

Iles, Chrissie, 2008, « Derek Jarman », dans Derek Jarman: Brutal Beauty, catalogue de l'exposition de la Serpentine Gallery (Londres, 23 février-13 avril 2008), Londres, Koenig Books, p. 64-76.

Jarman, Derek, 1984, Dancing Ledge, Londres, Quartet.

Lippard, Chris (dir.), 1996, By Angels Driven: the Films of Derek Jarman, Westport (Connecticut), Praeger.

Moholy-Nagy, László, [1930] (1934), « Problème du film moderne », dans Kristina Passuth, Moholy Nagy, Paris, Flammarion, p. 314-319.

Nash, Mark, 1985, « Innocence and Experience », Afterimage, $n^{\circ}$ 12, p. 30-35

O’Pray, Michael, 1985, « Derek Jarman's Cinema: Eros and Thanatos », Afterimage, n 12, p. 6-21.

-, 1996, Derek Jarman: Dreams of England, Londres, BFI Publishing.

Wollen, Roger (dir.), 1996, Derek Jarman: A Portrait, Londres, Thames and Hudson.

Wymer, Rowland, 2005, Derek Jarman, Manchester, Manchester University Press.

\section{Films de Derek Jarman cités (par ordre chronologique)}

The Art of Mirrors, 1973, 10 min, super-8.

Sebastiane, 1976, $86 \mathrm{~min}, 16 \mathrm{~mm}$.

Jubilee, 1978, $103 \mathrm{~min}, 16 \mathrm{~mm}$.

In the Shadow of the Sun, 1974-1981, 54 min, super-8 gonflé en 16 mm.

The Angelic Conversation, 1985, 81 min, mixed media.

Caravaggio, 1986, $93 \mathrm{~min}, 35 \mathrm{~mm}$.

The Last of England, 1987, 91 min, mixed media.

War Requiem, 1989, $86 \mathrm{~min}$, super-8 et $35 \mathrm{~mm}$.

The Garden, 1990, 92 min, mixed media.

Edward II, 1991, $91 \mathrm{~min}, 35 \mathrm{~mm}$.

Wittgenstein, 1992, $75 \mathrm{~min}, 16 \mathrm{~mm}$ et $35 \mathrm{~mm}$.

Blue, 1993, $76 \mathrm{~min}, 35 \mathrm{~mm}$. 


\section{NOTES}

1. "There We Are John...», interview de Derek Jarman par John Cartwright, réalisée par Ken McMullen en 1993, supplément du DVD The Last of England (Second Sight).

2. Jarman fut également décorateur (notamment pour les deux films de Ken Russell Les Diables / The Devils (1971) et Le Messie sauvage / Savage Messiah (1972) mais aussi pour des spectacles scéniques - théâtre, opéra et ballet) et l'auteur de plusieurs livres mêlant récits autobiographiques, notes de travail, réflexions philosophiques ou politiques et poésie. On trouvera une bibliographie complète des ouvrages de Jarman ainsi qu'une chronologie de son travail de décorateur dans Wollen (dir.) (1996).

3. Dès 1985, Mark Nash écrivait à ce propos : «Les films de Jarman sont élaborés à partir de tableaux, rappelant la peinture ou la sculpture [...]. La mise en scène, la direction artistique, l'image sont les qualités primordiales [...]. Le récit est secondaire» (Nash 1985: 32). Nous traduisons vers le français toutes les citations en anglais de l'article.

4. Les toiles citées dans Caravaggio par ordre d'apparition : Tête de Méduse, c. 1598, huile sur toile marouflée sur un bouclier en bois, diamètre $55 \mathrm{~cm}$, Offices, Florence ; Corbeille de fruits, c. 1601, huile sur toile, $31 \times 47 \mathrm{~cm}$, Pinacoteca Ambreosiana, Milan (que le jeune Caravaggio vend à un client) ; le Joueur de luth, c. 1595-1596, huile sur toile, $94 \times 119 \mathrm{~cm}$, Ermitage, Saint Petersbourg ; Autoportrait en Bacchus, c. 1593-1594, huile sur toile, $66 \times 52 \mathrm{~cm}$, Galleria Borghese, Rome ; Jeune homme portant une corbeille de fruits, 1593-1594, huile sur toile, $70 \times 67 \mathrm{~cm}$, Galleria Borghese, Rome ; Jeune garçon mordu par un lézard, 1593-1594, huile sur toile, $66 \times 49,5 \mathrm{~cm}$, National Gallery, Londres ; Musiciens, c. 1595, huile sur toile, $87,5 \times 115,9 \mathrm{~cm}$, Metropolitan Museum of Art, New York; Saint Jean-Baptiste, c. 1602 , huile sur toile, $129 \times 95 \mathrm{~cm}$, Pinacoteca Capitolina, Rome (référence indirecte lorsque Jerusalem caresse un bélier) ; Martyre de Saint Matthieu : première esquisse et tableau final, c. 1599-1600, huile sur toile, $322 \times 343 \mathrm{~cm}$, Saint-Louis-des-Français, Rome ; L'Amour vainqueur, c. 1601-1602, huile sur toile, $191 \times 148 \mathrm{~cm}$, Staaliche Museen, Berlin ; L'Incrédulité de Saint Thomas, c. 1602-1603, huile sur toile, $107 \times 146 \mathrm{~cm}$, Stiftung Schlosser une Garten, Sanssouci, Potsdam ; Saint Jean Baptiste, c. 1603-1605, huile sur toile, 173,4 ×132,1 cm, Nelson-Atkins Museuem of art, Kansas City; Madeleine repentante, 1593-1594, huile sur toile, 122,5 × 98,5 cm, Galleria Doria-Pamphilj, Rome ; Mort de la Vierge, c. 1601-1603, huile sur toile, $369 \times 245 \mathrm{~cm}$, musée du Louvres, Paris ; Saint Jérôme, c. 1605, huile sur toile, $112 \times 157 \mathrm{~cm}$, Galleria Borghese, Rome ; Mise au tombeau, c. 1602-1604, huile sur toile, $300 \times 203 \mathrm{~cm}$, Pinacoteca Vaticana, Rome. On peut en outre reconnaitre deux tableaux de deux autres peintres, le film jouant d'ailleurs d'anachronismes visuels et sonores, affichant par ce biais le parti pris de Jarman d'imposer sa vision personnelle et sa liberté créative à contre-courant de la minutie des reconstitutions historiques des heritage films: Marthe et Marie Madeleine de Bernadino Luini, c. 1516 , huile sur toile, $63,7 \times 85,5 \mathrm{~cm}$, San Diego Museum of Art (pour la robe de bal que porte Lena) et La Mort de Marat de Jacques-Louis David, 1793, huile sur toile, $165 \times 128 \mathrm{~cm}$, Musées royaux des beaux-arts de Belgique, Bruxelles (en clin d'œil ironique envers le critique Giovanni Baglione prenant son bain). Voir la liste des peintures du Caravage : http://fr.wikipedia.org/ wiki/Liste_des_peintures_du_Caravage.

5. C. 1598, huile sur toile marouflée sur un bouclier en bois, diamètre $55 \mathrm{~cm}$, Offices, Florence.

6. C. 1601-1602, huile sur toile, $191 \times 148$, Staatliche Museen, Berlin.

7. La toile en question est en fait la première esquisse du Martyre de saint Matthieu, mise en évidence par rayons $\mathrm{X}$.

8. C. 1599-1600, huile sur toile, $322 \times 343 \mathrm{~cm}$, Saint-Louis-des-Français, Rome.

9. Le film fut tourné en six semaines dans un entrepôt désaffecté dans les Docklands de Londres pour un budget de 475000 livres.

10. À la fin de Caravaggio, le peintre a pris la place du Christ dans la reconstitution de La Mise au tombeau. Dans The Garden (1990), la figure christique est tour à tour incarnée par un couple de 
jeunes hommes gay et une figure isolée d'homme mûr. Dans The Last of England, une gravure du Christ ainsi qu'un crucifix ornent les murs du studio de Jarman filmé endormi à son bureau.

11. Le directeur de la photographie Gabriel Beristain explique à ce propos que le parti pris esthétique était aussi lié à une contrainte technique, la pellicule Fuji utilisée ne pouvant pas reproduire parfaitement les noirs.

12. Le terme " acousmatique ", emprunté à Michel Chion, caractérise les sons entendus dont on ne voit pas la source.

13. Si le film Blue est indissolublement lié à l'avancée de la maladie du réalisateur, Jarman en a pourtant conçu l'idée dès 1974, après avoir vu une exposition des œuvres de Klein à la Tate. De même, à la suite de Klein, Jarman pose son film en acte de protestation contre ce qu'il appelle le "pandémonium visuel» («the pandemonium of image») qui vise en particulier le traitement médiatique des malades du sida à l'époque. Voir Wymer (2005 : 170-171).

\section{RÉSUMÉS}

En quoi l'œuvre de Derek Jarman peut-elle aider à penser les rapports entre peinture et cinéma au-delà de la simple citation? Partant du constat que les mêmes préoccupations informent la peinture et le cinéma de Jarman, cet article examine la manière dont la problématique picturale imprègne ses films, à travers en particulier deux questions fondamentales de l'histoire de l'art : d'une part celle de la dichotomie entre surface et profondeur, d'autre part celle d'une tension entre figuration et défiguration. Or, dans les deux cas, la question se pose en termes filmiques par le traitement de la lumière et des couleurs et se traduit par deux tendances apparemment opposées que sont l'épure et l'outrance. Ainsi le détour par la problématique picturale permet de montrer en quoi ces deux tendances sont constitutives d'une même poétique cinématographique, résolument anti-réaliste et soucieuse d'explorer les limites de la représentation. Dans les deux cas, le film est appréhendé comme support pictural à modeler - et non simplement comme support photographique à imprimer.

In what way can Derek Jarman's work help us question the relationships between painting and cinema beyond mere quotations? Given that both his pictorial and filmic works display the same thematic and formal preoccupations, this article examines how pictorial issues infuse his films, in particular through two main questions in art history, first the dichotomy between surface and depth, second, the tension between figuration and abstraction. In both cases, the questions are conveyed in filmic terms through the use of light and colour, resulting in two seemingly opposite aesthetic trends, from austere monochromes to flamboyant superimpositions and montage. The pictorial question thus enables us to understand how these two trends are part and parcel of the same filmic poetics, characterised by non-realism and a desire to explore the limits of representation. Film is no longer considered a mere photographic support to print but a genuine pictorial support to shape. 
INDEX

Mots-clés : Derek Jarman, cinéma britannique, poétique cinématographique, surface et profondeur, figuration et défiguration

Keywords : Derek Jarman, British cinema, film poetics, surface and depth, figuration and abstraction

\section{AUTEUR}

NICOLE CLOAREC

Université Rennes 1, LIDILE (EA 3874) 